\title{
The gap effect for eye and hand movements
}

\author{
HAROLD BEKKERING \\ University of Limburg, Maastricht, The Netherlands \\ and Max-Planck-Institute for Psychological Research, Munich, Germany \\ and \\ JAY PRATT and RICHARD A. ABRAMS \\ Washington University, St. Louis, Missouri
}

\begin{abstract}
A temporal gap between fixation point offset and stimulus onset typically yields shorter saccadic latencies to the stimulus than if the fixation stimulus remained on. Several researchers have explored the extent to which this gap also reduces latencies of other responses but have failed to find a gap effect isolated from general warning effects. Experiment 1, however, showed a robust gap effect for aimed hand movements (which required determination of a precise spatial location), regardless of whether the hand moved alone or was accompanied by a saccadic eye movement. Experiment 2 replicated this aimed hand gap effect and also showed a smaller effect for choice manual keypress responses (which required determination of the direction of response only). Experiment 3 showed no gap effect for simple manual keypress responses (which required no spatial determination). The results are consistent with an interpretation of the gap effect in terms of facilitation of spatially oriented responses.
\end{abstract}

Coordination between the oculomotor and manual motor systems is essential for successful interaction with our daily environment. However, a great deal of our knowledge about the control of these motor systems is based on results of experiments in which these systems have been studied separately. Evidence from several experiments requiring concurrent eye and hand movements suggests, however, that the oculomotor and manual motor systems are interrelated during the production of goal-directed movements to a visual target (e.g., Abrams, Meyer, \& Kornblum, 1990; Bekkering, Adam, Kingma, Huson, \& Whiting, 1994; Bekkering, Abrams, \& Pratt, 1995; Bekkering, Adam, Van den Aarssen, Kingma, \& Whiting, in press; Nemire \& Bridgeman, 1987). One useful approach to examining the relation between oculomotor and manual motor systems is to examine eye and hand movements using a paradigm that has been successfully applied to one of the motor systems. The present research was designed to explore the gap effect-a temporal gap between the fixation point offset and stimulus onset (known to have a robust influence on the oculomotor system)-on eye and hand responses.

This research was supported by Grant R29-MH45145 from the National Institutes of Health to R. A. A. and funding provided by the Netherlands Organization for Scientific Research (NWO) and the Foundation "De Drie Lichten" in the Netherlands to H. B. The authors thank J. Adam, J. Whiting, and two anonymous reviewers for helpful comments on this paper, and H. Korthase for excellent technical assistance. Correspondence should be sent to $\mathrm{H}$. Bekkering, Max-PlanckInstitute for Psychological Research, Leopoldstrasse 24, D-80802 München, Germany (e-mail: bekkering@mpipf-muenchen.mpg.de).

\section{The Gap Effect}

A temporal gap between the offset of a fixation point and the onset of a target stimulus results in shorter saccadic latencies than if the fixation point remained visible (see, e.g., Fischer \& Ramsperger, 1984; Reulen, 1984a, 1984b; Reuter-Lorenz, Hughes, \& Fendrich, 1991; Saslow, 1967). The reduced latencies produced by the advance offset of fixation have been referred to as the gap effect, and they represent an enhanced ability to initiate saccades beyond the mere warning benefits provided by the fixation offset. The effect appears to be most effective if the blank interval between fixation offset and target onset is approximately $200 \mathrm{msec}$. The saccadic latency reduction produced by the temporal gap has been attributed to oculomotor readiness (Saslow, 1967), facilitated sensory processing (e.g., Reulen, 1984a, 1984b), and covert attentional processes (e.g., Fischer, 1987; Fischer \& Weber, 1993). Recently, a good deal of research has suggested that the gap effect may consist of two components: a small general warning effect and a larger oculomotor-specific effect (Kingstone \& Klein, 1993a, 1993b; Reuter-Lorenz et al., 1991; Tam \& Ono, 1994; Tam \& Stelmach, 1993). This explanation for the gap effect assumes that during fixation, the oculomotor system effectively locks onto the fixation target and that eye movements toward new objects are inhibited (e.g., Guitton, Buchtel, \& Douglas, 1985). This bias against moving the eyes from a fixated object to a new target is independent of whether the actor is directing his/her attention to the fixated object or not (e.g., Kingstone \& Klein, 1993a, 1993b; Tam \& Stelmach, 1993; Walker, Kentridge, \& Findlay, 1995). Importantly, this oculomotor explanation suggests that the gap effect for a nonocular response should 
be small (due strictly to the warning effect) or not present at all.

For the most part, the prediction arising from the oculomotor explanation of small or negligible gap effects for manual responses has been confirmed when researchers have examined choice keypress responses. Iwasaki (1990) and Tam and Stelmach (1993) found evidence of a gap effect with choice manual keypress responses. Importantly, however, these studies did not include an auditory warning tone that coincided with the visual fixation offset. Without an auditory warning tone, it is difficult to determine whether the reduced latencies were due to the gap effect or to a generalized warning effect provided by the offset of the fixation point (e.g., Reuter-Lorenz, Oonk, Barnes, \& Hughes, 1995; Tam \& Stelmach, 1993). Using a paradigm that included such an auditory warning tone, Reuter-Lorenz et al. (1991) did not find a gap effect for choice manual keypress responses. Tam and Ono (1994) did not find a gap effect with choice manual keypress responses (even without a warning tone) either. Thus, the available evidence indicates that fixation offset does not benefit the manual motor system beyond general warning effects.

As noted, previous researchers examining manual gap effects have all used choice keypress responses (Iwasaki, 1990; Reuter-Lorenz et al., 1991; Tam \& Ono, 1994; Tam \& Stelmach, 1993). However, saccades and keypress responses differ in two important ways. First, unlike keypresses, saccades are aimed, target-directed, spatially oriented responses. That is, subjects making a saccadic response must localize the target in space and produce an aimed response toward the target. On the other hand, with keypress responses, subjects typically have their fingers already placed on the keys, and they need to simply press the correct key upon receipt of the target signal. The other difference between eye movements and keypresses is that saccades are mutually exclusive: One can make a saccade to the left or to the right but one cannot produce a saccade to go simultaneously to the left and right. Keypress responses are not mutually exclusive: One can easily press two keys simultaneously. These differences in the movements may reflect fundamental differences in the processes involved in planning and producing them. It may be that all aimed, mutually exclusive, spatially oriented movements are sensitive to the gap effect. If so, advance offset of fixation (i.e., a gap) might facilitate aimed hand movements. Alternatively, it is possible that the gap effect is strictly an oculomotor phenomenon. In that case, even spatially oriented hand movements would be expected to be unaffected by a gap.

\section{EXPERIMENT 1}

In Experiment 1, we explored the gap effect for manual responses. We used a manual response-a hand-pointing movement-as similar as possible to a saccadic response. Pointing responses are spatially oriented, aimed, and mutually exclusive. In the present experiment, subjects made aimed hand responses, saccadic eye movement responses, or both hand pointing and saccadic responses to a peripheral target (similar to the methodology of Bekkering et al., 1994). By using such a design, we sought to (1) replicate the gap effect when only saccadic responses were required, (2) determine whether the gap effect exists for hand-pointing responses, and (3) determine the impact, if any, of simultaneous eye and hand movements on the gap effect. To control for the general warning benefits provided by the offset of the fixation point, an auditory warning tone was always presented prior to the presentation of the target stimulus.

\section{Method}

Subjects. Twelve Washington University (St. Louis) students participated in an 80 -min session. All subjects were right-handed, did not wear corrective lenses, and were naive with respect to the purpose of the study. Each subject was paid $\$ 10$ for his/her participation.

Apparatus. An IBM AT computer controlled the presentation of stimuli and the acquisition of data throughout the experiment. The subject was seated at a table in a dark room with his/her eyes $38 \mathrm{~cm}$ from a cathode-ray tube (CRT) display. The subject's head was fixed by means of a dental impression plate. The left eye was occluded, and the position of the right eye was monitored with a scleralreflectance device (Applied Science Laboratories, Model 210) mounted on a spectacles frame. The subject's right elbow rested on a padded support on the table in front of him/her. Subjects produced pointing movements by means of a handle mounted directly in front of and below the CRT. A cardboard shield prevented the subjects from viewing their hand or the handle.

Data analysis. The analog output from the eye movement monitor was digitized at a rate of $1000 \mathrm{~Hz}$ with a resolution of $0.05^{\circ}$. Calibration of the monitor was accomplished by having the subject fixate at five evenly spaced points across the CRT. Eye position was then computed by using piecewise linear interpolation of the calibration points. Calibration was performed at the beginning of each session and verified before each trial (if verification failed, the subject had to recalibrate). Saccadic eye movements were detected by differentiating and filtering the signal obtained from the eye movement monitor with a low-pass digital filter $(80-\mathrm{Hz}$ cutoff). The resulting velocity profiles were analyzed to determine whether a saccade was produced. The beginning of a saccade was defined to be the first moment in time at which the velocity of the eye exceeded $10 \% \mathrm{sec}$ and remained above that value continously for at least $10 \mathrm{msec}$ while subsequently exceeding $35 \% \mathrm{sec}$. The end of the saccade was defined to be at the first moment in time afterward at which the velocity of the eye fell below $10 \% \mathrm{sec}$. Using this algorithm, we were able to detect the occurrence of saccades larger than about $0.7^{\circ}$ of visual angle.

The handle moved from side to side along a nearly frictionless track and was grasped in the right hand. A precision potentiometer attached to the track provided an analog signal indicating the position of the handle. This signal was digitized at a rate of $1000 \mathrm{~Hz}$ with a resolution of $0.01^{\circ}$ during the critical portions of each trial. The handle was calibrated at the beginning of each session. In order to detect the presence of hand movements, the signal obtained from the handle on each trial was differentiated and filtered using a low-pass digital filter ( $40-\mathrm{Hz}$ cutoff). The resulting velocity profiles were analyzed to determine whether a hand movement was produced. The beginning of a hand movement was defined to be the first moment in time at which the velocity of the handle exceeded $5^{\circ} / \mathrm{sec}$ and remained above that value continously for at least the next $20 \mathrm{msec}$.

Both eye and hand responses detected within an interval of $100 \mathrm{msec}$ after target onset were considered to be of anticipatory nature and were excluded from analysis. In the case of anticipatory responses, a central display was presented to the subjects with the sentence "Eye moved too soon" or "Hand moved too soon." 


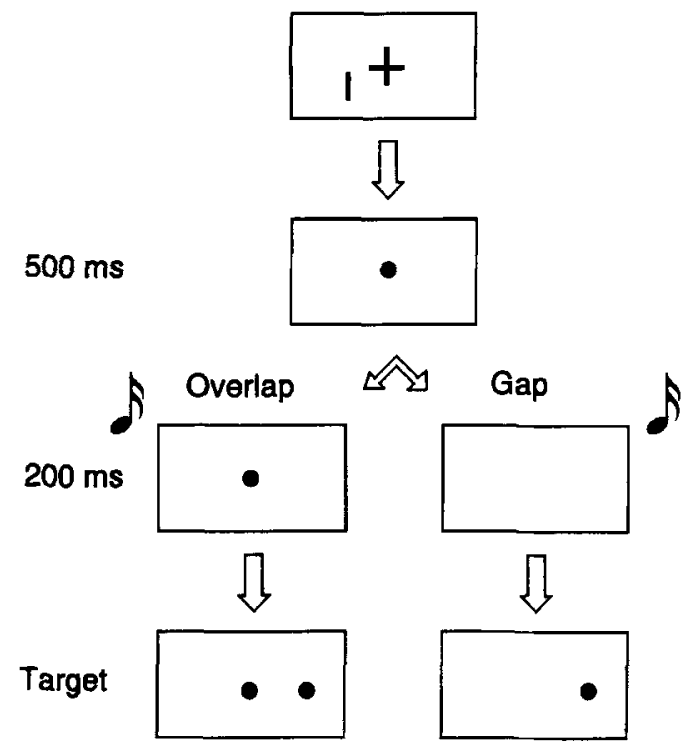

Figure 1. Sequence of events used in the present experiments. See the text for details.

Procedure. The sequence of events on a trial is presented in Figure 1. At the beginning of each trial, subjects moved the handle so that it was aligned with a plus sign (+) at $0^{\circ}$ (straight ahead). In order to facilitate this alignment, a small cursor appeared on the screen and moved in correspondence with the handle. Subjects were also required to fixate on the plus sign. Following both the alignment of the handle and successful fixation, the plus sign changed to a dot and the cursor was removed. Five hundred milliseconds later, an auditory warning signal was presented for $50 \mathrm{msec}$. In the gap condition, the dot was removed coincident with the offset of the warning signal. After an interval of $200 \mathrm{msec}$ (the gap), a peripheral target (a dot) appeared $10^{\circ}$ to the right or left of fixation. In the overlap condition, the dot at fixation remained visible for the $200-\mathrm{msec}$ interval following the warning tone offset, followed by the appearance of the target (in the same manner as in the gap condition). In both conditions the target dot remained visible until the end of the trial. In the overlap condition, the fixation dot also remained visible until the end of the trial.

Three different types of responses were studied: In the look-only condition, subjects made saccadic eye movements to the target but did not move the handle from the fixation location. In the pointonly condition, subjects moved the handle to the target but were not permitted to move their eyes from the fixation location. In the look-and-point condition, subjects moved both their eyes and the handle to the target. Subjects were instructed to make all of their responses as quickly and as accurately as possible. Eyes and hands were monitored in all conditions to ensure that subjects made only the response that was appropriate to the condition. Subjects were given feedback if they erroneously moved their eyes ("Eye moved") or the handle ("Hand moved"), depending on the condition.

Design. Subjects served in two blocks of 50 trials each for all three response conditions (the look-only, point-only, look-andpoint conditions). The order of conditions was counterbalanced between subjects. In each block, 25 gap and 25 overlap trials were randomly presented. Targets were equally likely to appear to the right or left of fixation.

\section{Results}

For purposes of the analysis, the conditions that required a single response (the look-only and point-only conditions) were termed "single-task," whereas the look- and-point condition was termed "dual-task." A 2 (single or dual task) $\times 2$ (eye or hand response) $\times 2$ (gap or overlap trial) $\times 2$ (right or left target) analysis of variance (ANOVA) was conducted on the latencies of eye and hand responses. These data appear in Figure 2 (collapsed over targets). Overall, eye movements were initiated sooner than were hand movements $[F(1,11)=59, p<$ $.0001]$.

Importantly, a main effect was found for trial type $[F(1,11)=34, p<.0001]$; gap trials $(M=284 \mathrm{msec})$ were faster than overlap trials $(M=316 \mathrm{msec})$. The task $\times$ trial type interaction was not significant $[F(1,11)<$ $1.3, p>.27$ ], indicating that the gap effect did not differ between the single- and dual-task conditions. However, the response $\times$ trial type interaction was significant $[F(1,11)=11, p<.005]$, with the gap effect being larger for eye movements $(M=42 \mathrm{msec})$ than for hand movements $(M=25 \mathrm{msec})$.

The error data appear in Table 1. Overall, $11.1 \%$ of the trials were excluded due to the errors. A 2 (response) $\times$ 2 (task) $\times 2$ (trial type) ANOVA was used to examine the error data, and no significant main effects or interactions were found $\left[F_{\mathrm{S}}(1,11)<3.5, p \mathrm{~s}>.05\right]$. Anticipation errors-reaction times (RTs) less than $100 \mathrm{msec}$-made up $21 \%$ of the total errors ( $1.2 \%$ of total trials), and a 2 (response) $\times 2$ (task) $\times 2$ (trial) ANOVA revealed no main effects or interaction effects $[F s(1,11)<4.0, p s>.05]$.

\section{Discussion}

The results of Experiment 1 clearly demonstrate that the gap effect facilitates the initiation of saccadic eye movements as well as the initiation of hand-pointing move-

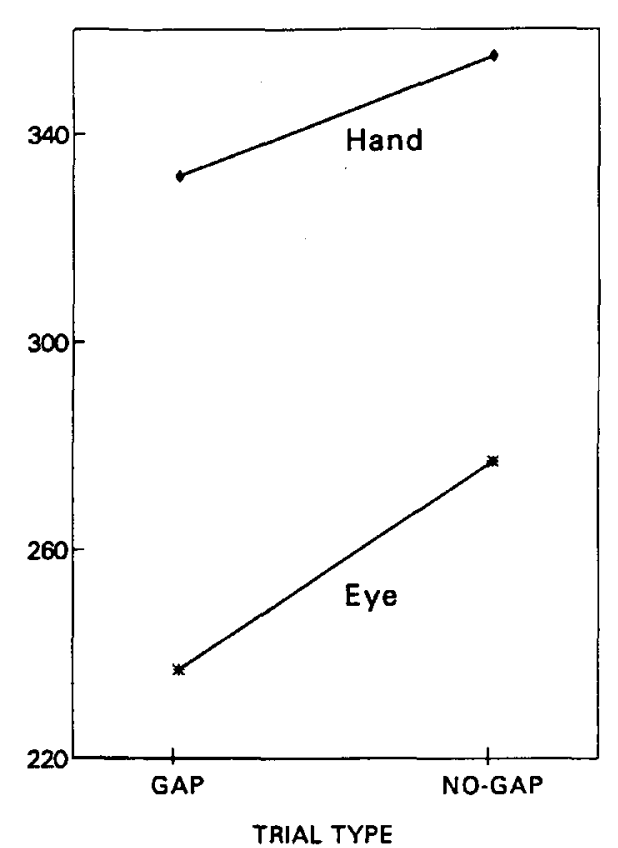

Figure 2. Mean latencies (in milliseconds) of saccadic eye and aimed hand movements in gap and overlap trials (Experiment 1). A robust gap effect is demonstrated for both eye and hand responses. 
Table 1

Percentage of Total Errors (TE) and Anticipation Errors (AE) on Gap and Overlap Trials for Each Experimental Condition

\begin{tabular}{|c|c|c|c|c|}
\hline \multirow[b]{3}{*}{ Condition } & \multicolumn{4}{|c|}{ Trials } \\
\hline & \multicolumn{2}{|c|}{ Gap } & \multicolumn{2}{|c|}{ Overlap } \\
\hline & $\%$ TE & $\% \mathrm{AE}$ & $\% \mathrm{TE}$ & $\% \mathrm{AE}$ \\
\hline \multicolumn{5}{|c|}{ Experiment 1} \\
\hline \multicolumn{5}{|l|}{ Eye } \\
\hline Look and point & 8.5 & 2.5 & 10.5 & 2 \\
\hline Look only & 11 & 2.2 & 10.2 & 3 \\
\hline \multicolumn{5}{|l|}{ Hand } \\
\hline Look and point & 8.5 & 3.8 & 9.8 & 2.2 \\
\hline Point only & 20.3 & 2.3 & 9.7 & 2.3 \\
\hline \multicolumn{5}{|c|}{ Experiment 2} \\
\hline Hand, point only & 15 & 1.8 & 8.7 & 1.5 \\
\hline Keypress, choice & 19 & 0.3 & 7.2 & 0.3 \\
\hline \multicolumn{5}{|c|}{ Experiment 3} \\
\hline \multicolumn{5}{|l|}{ Keypress } \\
\hline Choice & 4.7 & 0.4 & 3.7 & 0.5 \\
\hline Simple & 17 & 8.0 & 13.8 & 6.5 \\
\hline
\end{tabular}

ments. Moreover, the gap effect for hand movements was independent of the occurrence of eye movements. It seems unlikely that the gap effect found in this experiment can be attributed solely to a general warning effect of the visual offset, because an auditory warning signal was presented $200 \mathrm{msec}$ before the presentation of the target for both gap and overlap conditions. Thus, it appears that the gap effect is not limited to the oculomotor system. Additionally, hand latencies were not affected by simultaneous eye movements, whereas eye latencies were detrimentally affected by simultaneous hand movements. This pattern of results replicates the previous findings of Bekkering et al. (1994), who also found longer eye latencies in a look-and-point condition than in a look-only condition.

The size of the gap effect for both the eye and hand responses was unaffected by the presence or absence of simultaneous movement of the other system.

\section{EXPERIMENT 2}

The finding of a gap effect for hand-pointing movements is in contrast to the findings of several previous reports that failed to find gap effects in the manual motor system. However, as noted, these previous studies did not employ hand-pointing movements, but rather, choice keypress responses. It may be that the gap effect influences only responses that are spatially oriented and mutually exclusive. In order to examine that possibility, we studied a choice keypress response in the present experiment, in addition to the pointing movement from Experiment 1.

\section{Method}

Subjects. Twelve Washington University (St. Louis) students participated in a 50 -min session. All subjects were right-handed, did not wear corrective lenses, and were naive with respect to the purpose of the study. Each subject was paid \$7 for his/her participation. None had participated in the previous experiment.
Apparatus and Procedure. The apparatus and procedure were identical to those of Experiment 1. Two conditions were used in Experiment 2. One condition was the point-only condition from Experiment 1. In the other condition, the choice condition, subjects were required to press the " $z$ " key with their left hand in response to a target to the left of fixation and the "/" key with their right hand for a target to the right of fixation. Subjects were instructed to respond to the peripheral stimulus as quickly and as accurately as possible. The subject's eyes were monitored in both conditions to ensure that they did not look away from the fixation location.

Data analysis. Both hand and keypress responses that were detected within an interval of $100 \mathrm{msec}$ after target onset were considered to be anticipatory and were thus excluded from analysis. In the case of anticipatory responses, a central display was presented to the subjects with the sentence, "Hand moved too soon" or "Key pressed too soon." Trials on which eye movements were detected-using the criteria as described in Experiment 1-were also excluded from analysis. A central display was presented to the subjects with the sentence, "Eye moved."

Design. Subjects served in two blocks of 50 trials each for both conditions. Each condition included 25 gap and 25 overlap trials, all randomly presented. The order of conditions was completely counterbalanced across subjects. Targets were equally likely to appear to the left or right of fixation.

\section{Results}

Figure 3 shows latencies of aimed hand movements and choice manual keypress responses in gap and overlap trials (collapsed over targets). A 2 (point-only or choice condition) $\times 2$ (gap or overlap trial) $\times 2$ (right or left target) ANOVA was conducted on the data.

Overall, no difference in latencies were found between the point-only and choice conditions $[F(1,11)<3.25$, $p>.09]$. A main effect for trial type was found $[F(1,11)=$ $33, p<.0001]$, indicating a significant gap effect. A significant condition $\times$ trial type interaction $[F(1,11)=15$, $p<.003$ ] was found with the gap effect in the point-only condition ( $M=24 \mathrm{msec})$ that was larger than that for the choice condition $(M=11 \mathrm{msec})$. Paired, two-tailed $t$ tests indicated that there was a significant gap effect for both the point-only condition $[t(11)=5.98, p<.0001]$ and the choice condition $[t(11)=3.37, p<.006]$.

The error data appear in Table 1 . Overall, $12.2 \%$ of the trials were excluded due to errors. A 2 (response) $\times 2$ (trial type) ANOVA was used to examine the error data, and only a main effect for trial type was found $[F(1,11)=$ $16.3, p<.01]$, with more errors in gap trials than in overlap trials. Anticipatory errors (RTs of less than $100 \mathrm{msec}$ ) made up $8 \%$ of the total errors ( $1 \%$ of the total trials) and a 2 (response) $\times 2$ (trial type) ANOVA on the anticipation error data revealed a main effect for responses $[F(1,11)=$ $6.8, p<.05]$, but no other main effect or interaction effect $[F \mathrm{~s}(1,11)<1]$.

\section{Discussion}

Two major findings emerged from Experiment 2. First, a significant gap effect was again found for handpointing movements, replicating the results from Experiment 1 . Second, a smaller but significant gap effect was found for choice keypress responses. These two findings further suggest that the gap effect is not strictly an oculomotor phenomenon. However, the second result also sug- 


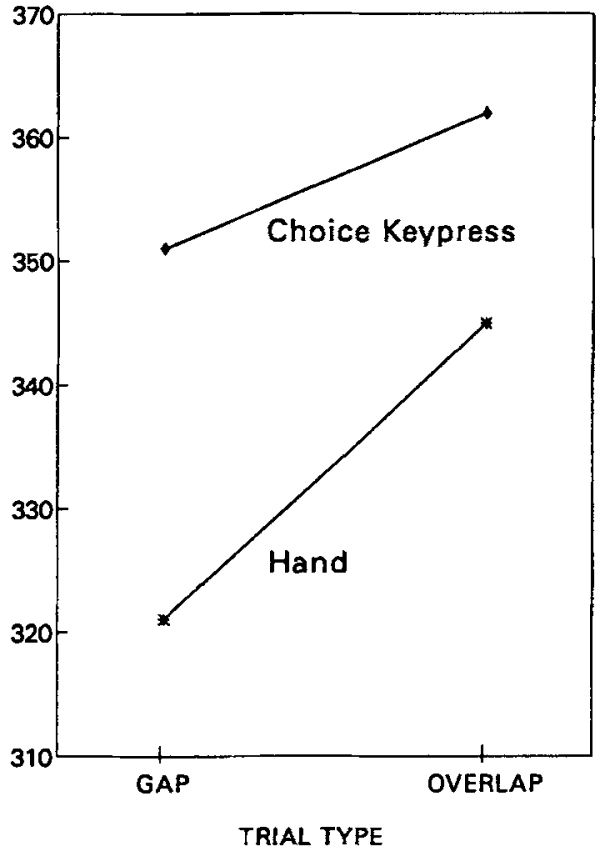

Figure 3. Mean latencies (in milliseconds) of aimed hand movements and choice manual keypress responses in gap and overlap trials (Experiment 2). A robust gap effect is demonstrated for hand responses and a small but reliable gap effect for choice manual keypress responses.

gests that mutually exclusive response alternatives are not necessary for a gap effect to occur. The finding of a gap effect for choice keypress responses opens the possibility that the reduced keypress latencies found by Iwasaki (1990) and Tam and Stelmach (1993) might not have been due solely to a generalized warning effect of a visual fixation offset, but instead may reflect a true gap effect. In the present experiments, the warning benefits of fixation offset were presumably also provided by the tone that was presented on all trials. As a result, we have assumed that any gap effect observed must reflect processes other than those involved in general alertness and warning effects. Nevertheless, it is possible that the warning available on gap trials (fixation offset plus tone) exceeded the warning benefits of the tone alone that was presented on the overlap trials. In order to examine this possibility, a third experiment was conducted.

\section{EXPERIMENT 3}

To test the possibility that the fixation offset served as a warning signal above and beyond the auditory warning signal, in Experiment 3 we compared choice keypress responses and simple keypress responses. If the visual offset does provide an additional warning benefit, then reduced latencies should be found in the gap condition for any response-including both types of keypress responses studied here.

\section{Method}

Subjects. Sixteen Washington University (St. Louis) students participated in a 50-min session. All subjects were right-handed, did not wear corrective lenses, and were naive with respect to the purpose of the study. Each subject was paid $\$ 7$ for his/her participation. None had taken part in the previous experiments.

Apparatus and Procedure. The apparatus and procedure were identical to those of Experiment 2. Two conditions were used in Experiment 3 . One condition was the choice condition from Experiment 2 . In the other condition, the simple condition, subjects were required to press the spacebar with their right hand when the target appeared (regardless of the location of the target). Subjects were instructed to respond to the target stimulus as quickly and accurately as possible. Subjects' eyes were monitored to ensure that they did not look away from the fixation location.

Data analysis. Choice and simple keypress responses detected within an interval of $100 \mathrm{msec}$ after target onset were considered to be anticipatory and thus were excluded from analysis. In the case of anticipatory responses, a central display was presented to the subjects with the sentence, "Key pressed too soon." Trials on which eye movements were detected-using the criteria as described in Experiment 1-were also excluded from analysis. A central display was presented to the subjects with the sentence, "Eye moved."

Design. Subjects served in two blocks of 50 trials each for both conditions. Each condition included 25 gap and 25 overlap trials, all randomly presented. The order of conditions was counterbalanced across subjects. Targets were equally likely to appear to the left or right of fixation.

\section{Results}

The latencies for the choice and simple conditions appear in Figure 4 (collapsed over targets). A 2 (choice or simple condition) $\times 2$ (gap or overlap trial) $\times 2$ (right or left target) ANOVA was conducted on the RTs.

Overall, a main effect for condition was found $[F(1,15)$ $=8.6, p<.01]$; responses in the choice condition $(M=$ $333 \mathrm{msec}$ ) were slower than those in the simple condition $(M=299 \mathrm{msec})$. No main effect for trial type was found $[F(1,15)<1]$. However, there was a significant condition $\times$ trial type interaction $[F(1,15)=5.3, p<.05]$, indicating a small gap effect for the choice condition $(6.3 \mathrm{msec})$ and none for the simple condition $(-4.3 \mathrm{msec})$. Twotailed, paired $t$ tests confirmed a significant gap effect in the choice condition $[t(15)=2.2, p<.05]$ but not in the simple condition $[t(15)<1]$.

The error data appear in Table 1. Overall, $14.6 \%$ of the trials were excluded due to the errors. A 2 (response) $\times 2$ (trial type) ANOVA was used to examine the error data, and only a main effect for trial type was found $[F(1,11)=$ $9, p<.01]$, with more errors in simple than in choice responses. Anticipation errors (RTs of less than $100 \mathrm{msec}$ ) made up $26 \%$ of the total errors ( $3.8 \%$ of the total trials), and a 2 (response) $\times 2$ (trial type) ANOVA on the anticipation error data revealed a main effect for response $[F(1,11)=6.8, p<.05]$ but no other main effect or interaction effect $\left[F_{s}(1,11)<1.5, p>.30\right]$.

\section{Discussion}

If the gap effect found in the previous experiments had been due to some general warning benefit provided by the fixation offset, both choice and simple conditions should 


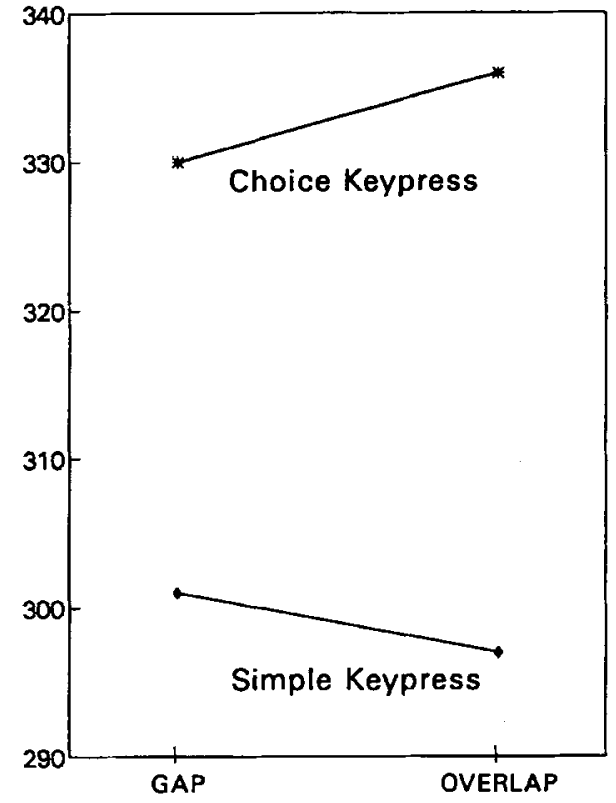

TRIAL TYPE

Figure 4. Mean latencies (in milliseconds) of choice manual keypress responses and simple manual keypress responses in gap and overlap trials (Experiment 3). A small but reliable gap effect is demonstrated for choice manual keypress responses and no gap effect at all for simple manual keypress responses.

have exhibited gap effects. This was not the case; only the choice condition showed a small but significant gap effect. Thus, it appears that only responses that have some goal-directed component exhibit gap effects. Although a choice keypress response has only a minimal goal-directed requirement (no precise localization of the target is required), a decision still has to be made with regard to direction. It appears that this minimum requirement of goal directness is critical for the gap effect in manual responses.

\section{GENERAL DISCUSSION}

\section{Mechanisms Underlying the Gap Effect}

The results of the present experiments, summarized in Table 2, shed some new light on the mechanisms underlying the gap effect. In particular, the gap effects for handpointing movements in Experiments 1 and 2 indicate that the facilitatory effect of fixation offset cannot be attributed to processes exclusively within the oculomotor system (e.g., Kingstone \& Klein, 1993a, 1993b). Moreover, the absence of a gap effect for simple manual keypress responses seems inconsistent with views that attribute the gap effect to attentional disengagement (see, e.g., Fischer, 1987; Fischer \& Breitmeyer, 1987; Mayfrank, Mobashery, Kimmig, \& Fischer, 1986). Attentional effects presumably occur early in visual processing (see, e.g., Hawkins, Shafto, \& Richardson, 1988) and are therefore likely to facilitate any type of response (see, e.g., Posner, 1980; Posner, Nissen, \& Ogden, 1978; Reuter-Lorenz et al., 1991). Additionally, the absence of a gap effect for simple manual keypress responses suggests that the gap effect is not solely a general warning or preparation effect (see, e.g., Kingstone \& Klein, 1993a, 1993b; Ross \& Ross, 1980, 1981). It might still be possible, however, that fixation offset on gap trials specific to oculomotor responses may have a residual warning effect (ReuterLorenz et al., 1995). Also, the fact that we found robust gap effects for both saccadic eye and hand-pointing movements, excluding responses with latencies of less than 100 msec, provides evidence for Kalesnykas and Hallett's notion (1987) that offset-onset effects are not due to anticipatory responses only.

The present findings further indicate that the nature of the response modulates the size of the gap effect. The gap effect was smallest for choice keypress responses, larger for hand pointing, and largest for saccadic eye movements. It is possible that the more precise the spatial requirements of the response, the greater facilitation provided by a fixation offset. The only response that was not spatial in nature, the simple keypress response from Experiment 3 , was also the only response for which a gap effect was not found.

It is not clear why we found significant gap effects in choice keypress responses when others have not. The present results suggest that the facilitated choice keypress latencies found with fixation offsets by Iwasaki (1990) and Tam and Stelmach (1993) might have been due to the gap effect or a warning signal effect. ReuterLorenz et al. (1991) and Tam and Ono (1994), however, failed to find any facilitation with a fixation offset for keypress response latencies. Nevertheless, a close inspection of the Reuter-Lorenz et al. and Tam and Ono data reveals faster choice RTs with fixation offset in both studies, although these facilitatory effects were not statistically significant. Both studies used relatively small numbers of subjects: 5 subjects in Reuter-Lorenz et al.'s

Table 2

Mean Latencies (RT, in Milliseconds) and Standard Deviations (SD) for Eye and Hand-Aiming Responses on Gap and Overlap Trials, and Gap Effects, for Each Experimental Condition

\begin{tabular}{|c|c|c|c|c|c|}
\hline \multirow[b]{3}{*}{ Condition } & \multicolumn{4}{|c|}{ Trials } & \multirow{3}{*}{$\begin{array}{c}\text { Gap } \\
\text { Effect }\end{array}$} \\
\hline & \multicolumn{2}{|c|}{ Gap } & \multicolumn{2}{|c|}{ Overlap } & \\
\hline & RT & $S D$ & RT & $S D$ & \\
\hline \multicolumn{6}{|c|}{ Experiment 1} \\
\hline \multicolumn{6}{|l|}{ Eye } \\
\hline Look and point & 254 & 52 & 292 & 39 & 38 \\
\hline Look only & 216 & 61 & 261 & 61 & 45 \\
\hline \multicolumn{6}{|l|}{ Hand } \\
\hline Look and point & 331 & 44 & 351 & 47 & 20 \\
\hline Point only & 333 & 36 & 359 & 40 & 26 \\
\hline \multicolumn{6}{|c|}{ Experiment 2} \\
\hline Hand, point only & 321 & 41 & 345 & 39 & 24 \\
\hline Keypress, choice & 351 & 39 & 362 & 37 & 11 \\
\hline \multicolumn{6}{|c|}{ Experiment 3} \\
\hline \multicolumn{6}{|l|}{ Keypress } \\
\hline Choice & 330 & 35 & 336 & 34 & 6 \\
\hline Simple & 301 & 63 & 297 & 51 & -4 \\
\hline
\end{tabular}


Experiment 1 and 5 subjects each in Tam and Ono's Experiments 2 and 3 . It may be that the relatively small number of subjects rendered these studies somewhat less sensitive to gap effects. Supporting this notion is the fact that the choice keypress latencies in Tam and Ono's Experiment 2 were $17 \mathrm{msec}$ faster with a fixation offset, an effect size larger than the two significant choice keypress gap effects found in the present experiments, which used $12(11 \mathrm{msec})$ and 16 subjects $(6.3 \mathrm{msec})$, respectively.

\section{Role of the Superior Colliculus in the Gap Effect}

Accumulating neurophysiological evidence suggests that the superior colliculus (SC) forms part of a system that facilitates active visual fixation (see, e.g., Munoz \& Wurtz, 1992, 1993a, 1993b) and that the SC is essential for the generation of short latency saccades that are produced in the gap paradigm (e.g., Munoz \& Wurtz, 1992; Schiller, Sandell, \& Maunsell, 1987). Additionally, Goldberg, Bushnell, and Bruce (1986) demonstrated that the threshold current needed to elicit a saccade from the SC increases during active fixation. The contribution of the $\mathrm{SC}$ to the gap effect is also consistent with the finding that the facilitatory effect appears to be limited to prosaccade, not antisaccade, latencies. This is because the SC does not appear to control responses other than saccadic eye movements toward a desired object (see, e.g., Reuter-Lorenz et al., 1991).

At first, the present findings of a gap effect for aimed hand movements seem to be in conflict with an interpretation of the gap effect as based on activity in the SC. However, Werner (1993) has recently reported neuronal activity in the primate $\mathrm{SC}$ before and during arm movements to visual targets. Werner further noted, "It is likely that the primate superior colliculus is not only involved in the initiation and control of orienting movements of the eyes but also in reaching movements of the arms" (p. 335). It might be that the SC is involved in all types of orienting movements toward external targets because it receives convergent input from visual, auditory, and somatosensory cortical areas. Consistent with this interpretation is the finding that projections have been found from the arm representation area of motor and premotor cortex to deep layers of the SC (Fries, 1984, 1985). However, it may be that we found larger gap effects for saccadic eye responses than for aimed hand responses because inhibitory effects of active fixation are more closely linked to orienting movements of the ocular motor system than to orienting movements of the hand motor system. Nevertheless, the present experiments clearly show that the assumed inhibition is not limited to the oculomotor system.

Additional work will be needed to further elucidate the complex interrelations between the various orienting and movement production systems, and the role that the $\mathrm{SC}$ may play in those behaviors. We believe that the present work represents a step in that direction.

\section{REFERENCES}

Abrams, R. A., Meyer, D. E., \& Kornblum, S. (1990). Eye-hand coordination: Oculomotor control in rapid aimed limb movements.
Journal of Experimental Psychology: Human Perception \& Performance, 14, 428-443.

BekKering, H., Abrams, R. A., \& Pratt, J. (1995). Transfer of saccadic adaptation to the manual motor system. Human Movement Science, 14, 155-164.

Bekkering, H., Adam, J. J., Kingma, H., Huson, A., \& Whiting, H. T. A. (1994), Reaction time latencies of eye and hand movements in single and dual-task conditions. Experimental Brain Research, 97, 471-476.

Bekkering, H., Adam, J. J., VAN den Aarssen, A., Kingma, H., \& WhITING, H. T. A. (in press). Interference effect between the initiation of saccadic eye and goal-directed hand movements. Experimental Brain Research.

Fischer, B. (1987). The preparation of visually guided saccades. Reviews of Physiology, Biochemistry \& Pharmacology, 106, 1-35.

FISCHER, B., \& BREITMEYER, B. (1987). Mechanisms of visual attention revealed by saccadic eye motions. Neuropsychologica, 25, 73-83.

FISCHER, B., \& RAMSPERGER, E. (1984). Human express saccades: Extremely short reaction times of goal-directed eye movements. $E x$ perimental Brain Research, 57, 191-195.

FISCHER, B., \& WEBER, H. (1993). Express saccades and visual attention. Behavioral \& Brain Sciences, 16, 553-610.

FRIES, W. (1984). Cortical projections to the superior colliculus in the macaque monkey: A retrograde study using peroxidase. Journal of Computative Neurology, 230, 55-76.

FRIES, W. (1985). Inputs from the motor and premotor cortex to the superior colliculus in the rhesus monkey. Behavioural Brain Research, 18, 95-105.

Goldberg, M. E., Bushnell, M. C., \& Bruce, J. C. (1986). The effect of attentive fixation on eye movements evoked by electrical stimulation of the frontal eye fields. Experimental Brain Research, 61, 579-584.

Guitton, D., Buchtel, H. A., \& Douglas, R. M. (1985). Frontal lobe lesions in man cause difficulties in suppressing reflexive glances and in generating goal-directed saccades. Experimental Brain Research, 58, 455-472.

Hawkins, H. L., Shafto, M. G., \& Richardson, K. (1988). Effects of target luminance and cue validity on the latency of visual detection. Perception \& Psychophysics, 44, 484-492.

IWASAKI, S. (1990). Facilitation of reaction times with gap paradigm: Comparison of manual and saccadic responses. Ergonomics, 33, 833-850.

Kalesnykas, R. P., \& Hallett, P. E. (1987). The differentation of visually guided and anticipatory saccades in gap and overlap paradigms. Experimental Brain Research, 68, 115-121.

KingSTONE, A., \& KLEIN, R, (1993a). Visual offset facilitates saccadic latency: Does pre-disengagement of visuo-spatial attention mediate this gap effect? Journal of Experimental Psychology: Human Perception \& Performance, 19, 1251-1265.

KIngSTONE, A., \& KLEIN, R. (1993b). What are human express saccades? Perception \& Psychophysics, 54, 260-273.

Mayfrank, L., Mobashery, M., Kimmig, H., \& Fischer, B. (1986). The role of fixation and visual attention in the occurrence of express saccades in man. European Archives of Psychiatry \& Neurological Sciences, 235, 269-275.

Munoz, D. P., \& WuRTz, R. H. (1992). Role of the rostral superior colliculus in active fixation and execution of express saccades. Journal of Neurophysiology, 67, 1000-1002.

Munoz, D. P., \& WuRTz, R. H. (1993a). Fixation cells in monkey superior colliculus I. Characteristics of cell discharge. Journal of Neurophysiology, 70, 559-575.

Munoz, D. P., \& WURTZ, R. H. (1993b). Fixation cells in monkey superior colliculus II. Reversible activation and deactivation. Journal of Neurophysiology, 70, 576-589.

NEMIRE, K., \& BRIDGEMAN, B. (1987). Oculomotor and skeletal motor systems share one map of visual space. Vision Research, 27, 393-400.

POSNER, M. I. (1980). Orienting of attention. Quarterly Journal of Experimental Psychology, 32, 3-25.

Posner, M. I., Nissen, M. J., \& OGden, W. C. (1978). What is the chapter title? In H. L. Pick \& I. J. Saltzman (Eds.), Attended and unattended processing modes: The role of set for spatial location. Models of perceiving and information processing (pp. 137-157). Hillsdale, $\mathrm{NJ}$ : Erlbaum. 
REULEN, J. P. H. (1984a). Latency of visually evoked saccadic eye movements: 1. Saccadic latency and the facilitation model. Biological Cybernetics, 50, 251-263.

REULEN, J. P. H. (1984b). Latency of visually evoked saccadic eye movements: II. Temporal properties of the facilitation mechanism. Biological Cybernetics, 50, 263-271.

Reuter-Lorenz, P. A., Hughes, H. C., \& Fendrich, R. (1991). The reduction of saccadic latency by prior offset of the fixation point: An analysis of the gap effect. Perception \& Psychophysics, 49, 167-175.

Reuter-Lorenz, P. A., OONK, H. M., Barnes, L. L.. \& Hughes, H. C. (1995). Effects of warning signals and fixation point offsets on the latencies of pro- versus antisaccades: Implications for an interpretation of the gap effect. Experimental Brain Research, 103, 287-293.

Ross, L. E., \& Ross, S. M. (1980). Saccade latency and warning signals: Stimulus onset, offset, and change as warning events. Perception \& Psychophysics, 27, 263-27!

Ross, L. E., \& Ross, S. M. (1981). Saccade latency and warning signals: Effects of auditory and visual stimulus onset and offset. Perception \& Psychophysics, 29, 429-437.

SASLOW, M. G. (1967). Latency for saccadic eye movement. Journal of the Optical Society of America, 57, 1030-1033.
SChIller, P. H., SANDeld, J. H., \& MaUnsell, J. H. R. (1987). The effect of frontal eye field and superior colliculus lesions on saccadic latencies in the rhesus monkey. Journal of Neurophysiology, 57, 1033-1049.

TAM, W., \& ONO, H. (1994). Fixation disengagement and eyemovement latency. Perception \& Psychophysics, 56, 251-260.

TAM, W., \& StelmaCH, L. (1993). Viewing behavior: Ocular and attentional disengagement. Perception \& Psychophysics, 54, 211222.

WaLKer, R., Kentridge, R. W., \& Findlay, J. M. (1995). Independent contributions of the orienting of attention, fixation offset and bilateral stimulation on human saccadic latencies. Experimental Brain Research, 103, 294-310.

WERNER, W, (1993). Neurons in the primate superior colliculus are active before and during arm movements to visual targets. European Journal of Neuroscience, 5, 335-340.

(Manuscript received December 7, 1994; revision accepted for publication August 28, 1995.) 